\title{
Pensamento Computacional Desplugado: Ensino e Avaliação na Educação Primária da Espanha
}

\author{
Christian P. Brackmann ${ }^{1}$, Rafael M. Boucinha ${ }^{2}$, \\ Marcos Román-González ${ }^{3}$, Dante Barone ${ }^{2}$, Ana Casali ${ }^{4}$ \\ ${ }^{1}$ Instituto Federal Farroupilha (IFFAR), Santa Maria, RS, Brasil \\ ${ }^{2}$ Universidade Federal do Rio Grande do Sul (UFRGS), Porto Alegre, RS, Brazil \\ ${ }^{3}$ Universidad Nacional de Educación a Distancia (UNED), Madrid, CM, Espanha \\ ${ }^{4}$ Universidad Nacional de Rosario (UNR), Rosario, SF, Argentina \\ brackmanneiffarroupilha.edu.br, rmboucinhaegmail.com, \\ mroman@edu.uned.es, barone@inf.ufrgs.br, acasali@fceia.unr.edu.ar
}

\begin{abstract}
Computational Thinking (CT) has been placing the focus of educational innovation as a set of troubleshooting skills. Unfortunately, there is not a consensus if the teaching methodology and the available materials attend the expectations of the lecturers. To prove the impact that CT training has in primary school, we attempted to evaluate Spanish students with a QuasiExperimental approach to prove if the activities are effective for students who live in areas where there are no electronic devices, Internet or even electrical power can be also benefited. The results show statistically significant improvement. In this way, we are able to reinforce the claim that CS unplugged is an effective approach for students who live in unprivileged areas.
\end{abstract}

Resumo. O Pensamento Computacional (PC) vem gerando um novo foco educacional nas escolas mundiais como um conjunto de aptidões para a solução de problemas. Até o momento, não há um consenso de metodologia de ensino e disponibilidade de material para atender as expectativas dos professores. Para sanar essa incerteza, realizou-se uma avaliação de estudantes da educação primária espanhola com uma abordagem QuaseExperimental no intuito de beneficiar crianças em regiões/escolas onde não há dispositivos eletrônicos, Internet e até mesmo energia elétrica. Os resultados apresentam dados estatísticos que comprovam uma melhoria significativa no desempenho dos estudantes que tiveram atividades de PC Desplugado.

\section{Introdução}

Vivemos em tempos marcados pela fluidez da informação e pela valorização do conhecimento. Mais do que nunca, para lidar com a informação, processá-la e transformála em aptidões para a vida, exige-se, em primeiro lugar, o domínio de uma série de ferramentas e recursos tecnológicos que devem ser acessíveis a todos, sem distinção de qualquer natureza. Nos tempos atuais, o desafio que se impõe aos usuários é criar seus próprios sistemas (por exemplo, programas e jogos) ou modificar os existentes de acordo com sua necessidade pessoal. É neste contexto que surge a habilidade que é vista como crucial no século 21: o Pensamento Computacional (PC) (Kologeski et al., 2016). Devido a essa tendência mundial, o PC vem sendo adotado em escolas da educação básica em diversos países (Brackmann et al., 2016). 
Wing (2006) define o PC como uma atividade mental para a formulação de um problema que é possível ser resolvido computacionalmente, ou seja, processos de pensamentos envolvidos na identificação de um problema e expressar a sua solução de forma eficaz, de tal forma que uma máquina ou uma pessoa possa executar. O PC utiliza quatro dimensões (também conhecido como pilares) para atingir o objetivo principal dessa abordagem: solução de problemas. Pesquisas lideradas pela Code.Org (2015), Liukas (2015) e BBC Learning (2015) mesclaram os elementos citados por Grover e Pea (2013) e resumiram nos chamados "Quatro Pilares do Pensamento Computacional", sendo eles: Decomposição, Reconhecimento de Padrões, Abstração e Algoritmos. Todos os Quatro Pilares têm grande importância e são interdependentes durante o processo de formulação de soluções computacionalmente viáveis.

O Pensamento Computacional envolve identificar um problema complexo e quebrá-lo em pedaços menores e mais fáceis de gerenciar (Decomposição). Cada um desses problemas menores pode ser analisado individualmente com maior profundidade, identificando problemas parecidos que já foram solucionados anteriormente (Reconhecimento de padrões), focando apenas nos detalhes que são importantes, enquanto informações irrelevantes são ignoradas (Abstração). Por último, passos ou regras simples podem ser criados para resolver cada um dos subproblemas encontrados (Algoritmos). Seguindo os passos ou regras utilizadas para criar um código, é possível também ser compreendido por sistemas computacionais e, consequentemente, utilizado na resolução de problemas complexos de forma eficiente, independentemente da carreira profissional que o estudante deseja seguir.

Neste contexto, foram criadas e adaptadas diferentes atividades para professores e alunos de maneira que possam usar e replicar esse material para suas aulas sem a necessidade de equipamentos eletrônicos, Internet ou eletricidade para que crianças possam estudar conceitos da Computação em escolas que não possuem equipamentos apropriados (e.g. estragados, ultrapassados ou na ausência destes) ou localizadas em áreas geográficas distantes (e.g. áreas rurais ou florestais). Acredita-se que com o uso dessas atividades desplugadas (sem a necessidade de máquinas) é possível ensinar Pensamento Computacional de maneira mais acessível, ou seja, usando basicamente papel, tesoura, canetas, lápis de colorir, cola e demais materiais escolares de uso comum.

$\mathrm{Na}$ literatura é raro encontrar pesquisas a respeito da aplicação e avaliação de estudantes de maneira desplugada. Para preencher esse vazio, o presente trabalho apresenta uma pesquisa realizada em duas escolas primárias na Espanha para avaliar se as crianças têm uma alteração no desempenho das habilidades relacionadas ao Pensamento Computacional através de atividades sem computadores. Sendo assim, esta pesquisa tem como objetivo verificar se aulas de Pensamento Computacional Desplugado na educação primária são eficazes através da aplicação de um pré e pós teste, realizados antes e após as aulas de PC Desplugado.

\section{Contextualização}

Muitos tópicos importantes da Ciência da Computação (CC) podem ser ensinados sem o uso de computadores. A abordagem desplugada da $\mathrm{CC}$ introduz conceitos de hardware e software que impulsionam as tecnologias cotidianas até pessoas não-técnicas. Em vez de participar de uma aula expositiva, as atividades desplugadas ocorrem frequentemente através da aprendizagem cinestésica (e.g. movimentar-se, usar cartões, cortar, colar, desenhar, pintar, resolver enigmas, etc.) e os estudantes trabalham entre si para aprender conceitos da CC. 
O surgimento do Pensamento Computacional Desplugado não é muito claro, pois a necessidade de abstração para a criação de qualquer software e hardware é essencial. É importante constar também que o uso de exemplos físicos e materiais escolares são comuns para simular o comportamento de máquinas até os dias atuais em cursos de graduação. Quando se trata de salas de aulas da educação básica, os primeiros registros são encontrados a partir de 1997 quando Bell et al. (1997) lançou um rascunho de um livro em formato digital denominado "Computer Science Unplugged... Off-line activities and games for all ages" para professores de todos os níveis escolares que queriam dar um diferencial a seus alunos. A ideia foi muito bem recebida pelos demais professores e a própria academia. Devido à qualidade do material publicado, a Association for Computing Machinery (ACM) recomendou que as atividades contidas no livro fizessem parte do currículo proposto pela Computer Science Teachers Association (CSTA) dos Estados Unidos da América (Association for Computing Machinery, 2003). Até a publicação deste artigo, o livro CS Unplugged se encontra na versão 3.1 e pode ser acessado no site do projeto (Bell et al., 2015).

Existem diversos estudos que investigam a eficiência de linguagens de programação (visual e código) com crianças Román-Gonzáles et al. (2015), Román-Gonzáles (2016), Grover et al. (2017), Franklin et al. (2017), porém carece de abordagens desplugadas. Outras pesquisas tentam padronizar a avaliação e o ensino de atividade de PC Desplugado, como por exemplo: 1) Nishida et al. (2009) onde apresenta uma proposta de padrão de design, 2) uma avaliação do PC de maneira transversal em uma escola de ensino médio (Feaster et al., 2011); 3) estudos de casos no processo de adoção do PC na sala de aula (Curzon, 2013); 4) avaliação dos pontos de vista de estudantes a respeito da CC antes e após aulas de PC (Taub et al., 2009); e também 5) sugestões de como professores podem avaliar o progresso dos estudantes ao realizar atividades de PC (Curzon et al., 2014). Lambert et al. (2009) realizou uma tentativa similar, porém na identificação de um aumento no interesse nas áreas da Computação ou Matemática, sem verificar um aumento/decremento nas habilidades relacionadas ao Pensamento Computacional.

A solução proposta por Rodriguez et al. (2017) procura avaliar estudantes com atividades desplugadas basicamente em três níveis (proficiente, parcialmente proficiente e insatisfatório) ou Campos et al. (2014) que também tenta a adoção de um teste, porém não possui um resultado satisfatório. Já Pasqueline et al. (2012) realiza uma avaliação de acertos de estudantes, sem o uso de pré e pós-teste para verificar alterações no desempenho dos mesmos.

Os trabalhos aqui citados são parte de um grande conjunto de pesquisas que tentam medir as habilidades do PC, porém não utilizam uma solução direta, de fácil aplicação e com um processo de validação formal para que se possa atingir um resultado mais preciso como proposto por Román-González (2016).

A revisão de estudos que fornecem evidências sobre a utilidade da computação desplugada para desenvolver habilidades do PC mostra que ainda há necessidade de nova pesquisas empíricas sobre esse assunto, especialmente quando se trata de seu uso em escolas primárias. Consequentemente, com a investigação relatada neste artigo, tenta-se esclarecer um pouco mais a respeito desse assunto.

\section{Materiais e Métodos}

Nesta seção apresenta-se o material desenvolvido e adaptado, dos participantes envolvidos e do processo de execução da pesquisa. 
VI Congresso Brasileiro de Informática na Educação (CBIE 2017)

Anais dos Workshops do VI Congresso Brasileiro de Informática na Educação (WCBIE 2017)

\subsection{Participantes e Grupos de Teste}

Os testes e as aulas de PC foram aplicados em um total de 72 alunos dos quintos e sextos anos do ensino fundamental, meninos e meninas da rede pública de ensino na cidade de Madrid, Espanha. Nenhum dos participantes tinha uma experiência de programação formal. A distribuição dos participantes por gênero, nível, idade e grupo são apresentados na Tabela 1 .

Tabela 1. Distribuição dos sujeitos participantes da pesquisa

\begin{tabular}{lcccccc}
\hline & \multirow{2}{*}{ Ano } & \multirow{2}{*}{ Idade } & \multirow{2}{*}{ Grupo } & \multicolumn{2}{c}{ Sexo } & \multirow{2}{*}{ Total } \\
\cline { 5 - 6 } & & & Masculino & Feminino & \multirow{2}{*}{ Escola A } \\
\cline { 5 - 6 } & 5 & $10-11$ anos & Controle & 10 & 13 & 23 \\
\hline Escola A & 5 & $10-11$ anos & Experimental & 11 & 8 & 19 \\
\hline Escola B & 6 & $11-12$ anos & Controle & 6 & 8 & 14 \\
\hline Escola B & 6 & $11-12$ anos & Experimental & 9 & 7 & 16 \\
\hline & & Total & 36 & 36 & 72 \\
\hline
\end{tabular}

\subsection{Instrumento Avaliativo: Teste de Pensamento Computacional}

O teste utilizado na pesquisa para medir o desenvolvimento das habilidades que fazem parte do Pensamento Computacional foi desenvolvido pelo pesquisador espanhol RománGonzález et al. (2015). Este teste tenta identificar a habilidade de formação e solução de problemas, baseando-se nos conceitos fundamentais da Computação, além de utilizar sintaxes lógicas usadas nas linguagens de programação. É o único teste encontrado na literatura com essas características. As questões que compõem o instrumento avaliativo incluem conceitos dos quatro pilares do Pensamento Computacional: abstração, decomposição, reconhecimento de padrões e algoritmos. Devido ao fato de o teste ter passado por um rigoroso processo de validação, o autor entende que os resultados são confiáveis (Román-González, 2015). O instrumento é composto por 28 questões de múltipla escolha, sendo que cada questão possui quatro alternativas de resposta e somente uma é válida. Um exemplo de questão é apresentado na Figura 1. Sua aplicação ocorre em navegadores (e.g. Chrome, Firefox, Edge) e pode ser acessado de qualquer dispositivo. Nesta pesquisa utilizaram-se exclusivamente os computadores do laboratório de informática das escolas.

\subsection{Desenvolvimento das Atividades}

A maior parte dos materiais desenvolvidos foram criados e os demais foram traduzidos para o Espanhol e adaptados de atividades pré-existentes, como por exemplo o livro "Hello Rubby" (Liukas, 2015) e o jogo de tabuleiro "Code Master", criado por Engelberg et al. (2015). Na tentativa de atrair a atenção das crianças, foram também utilizados personagens populares brasileiros nas atividades. Para que o leitor se familiarize com as atividades trabalhadas, são apresentadas algumas delas no Quadro 1. É importante citar que as atividades têm um objetivo principal de auxiliar no processo de ensinoaprendizagem dos conceitos da Computação para crianças da educação básica. Sua aplicação é melhor descrita na seção seguinte. 
VI Congresso Brasileiro de Informática na Educação (CBIE 2017)

Anais dos Workshops do VI Congresso Brasileiro de Informática na Educação (WCBIE 2017)

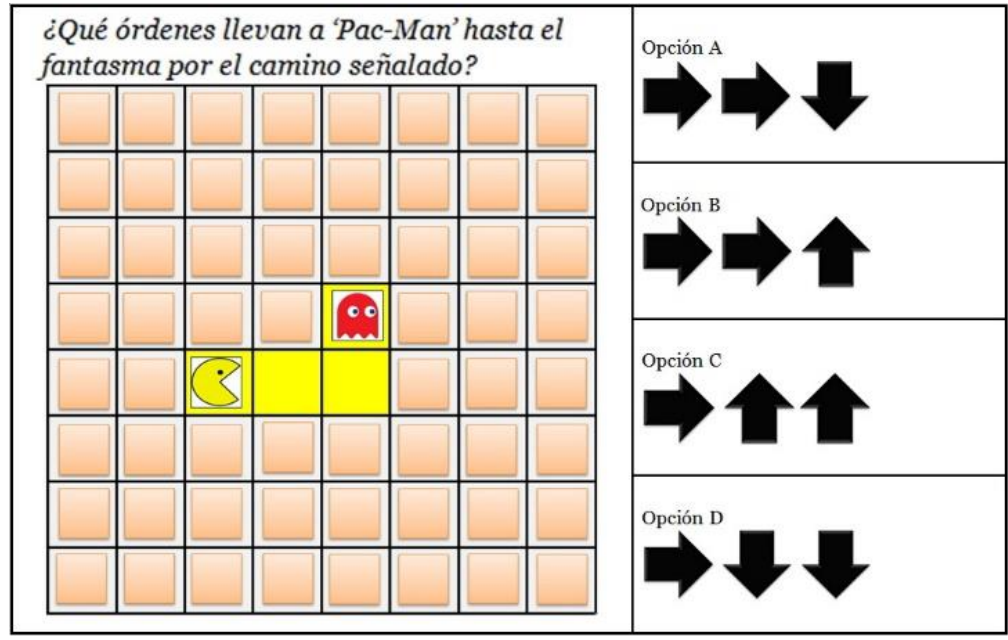

Figura 1. Exemplo de questão do Teste de Pensamento Computacional: "Qual sequência leva o 'Pac-Man' até o fantasma pelo caminho indicado?”

\section{Quadro 1 - Exemplos de atividades utilizadas nas intervenções}

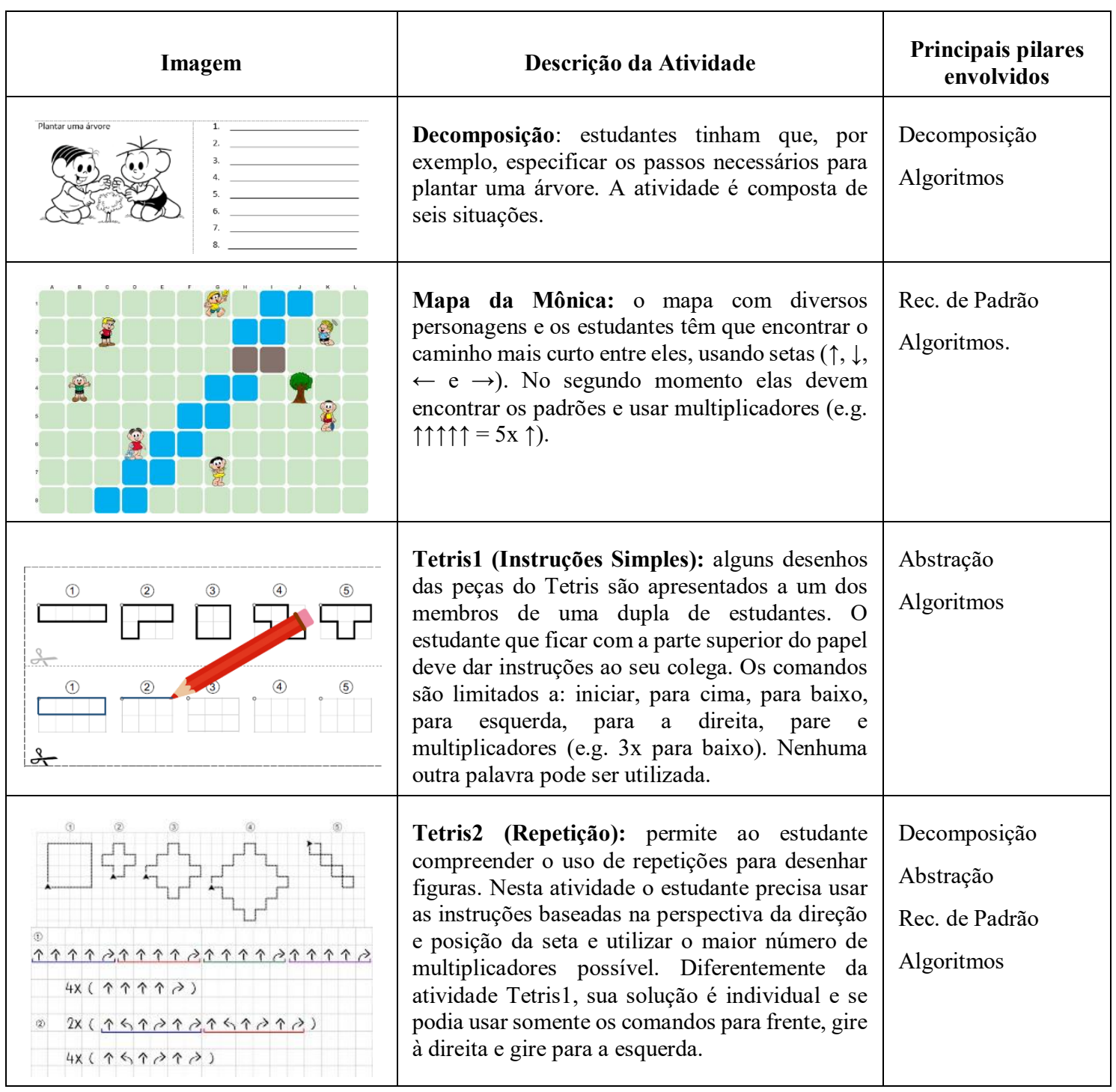


VI Congresso Brasileiro de Informática na Educação (CBIE 2017)

Anais dos Workshops do VI Congresso Brasileiro de Informática na Educação (WCBIE 2017)

\begin{tabular}{|c|c|c|}
\hline Imagem & Descrição da Atividade & $\begin{array}{c}\text { Principais pilares } \\
\text { envolvidos }\end{array}$ \\
\hline 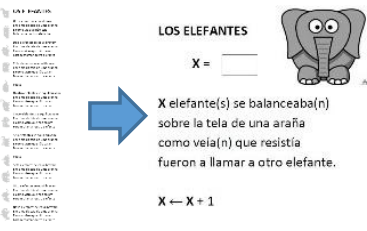 & $\begin{array}{l}\text { Os Elefantes: utiliza uma música popular entre } \\
\text { as crianças para exemplificar como uma canção } \\
\text { pode se tornar um algoritmo. Nesta música em } \\
\text { especial são trabalhados os conceitos de } \\
\text { repetição, variáveis e condicionais. }\end{array}$ & $\begin{array}{l}\text { Rec. de Padrão } \\
\text { Algoritmos }\end{array}$ \\
\hline 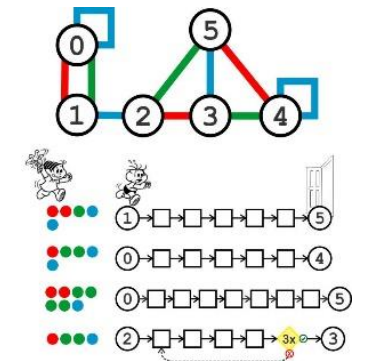 & $\begin{array}{l}\text { Rota de fuga do Cebolinha e Cascão: uma } \\
\text { versão mais simplificada do jogo de tabuleiro } \\
\text { Code Master desenvolvido pela companhia } \\
\text { ThinkFun (Engelberg et al., 2015). Nesta } \\
\text { atividade o estudante deve encontrar uma rota } \\
\text { entre dois nodos utilizando apenas as cores } \\
\text { permitidas para cada caminho. A atividade é } \\
\text { composta por quatro mapas e } 16 \text { desafios (rotas). }\end{array}$ & $\begin{array}{l}\text { Decomposição } \\
\text { Abstração } \\
\text { Algoritmos }\end{array}$ \\
\hline
\end{tabular}

\subsection{Procedimento}

As escolas foram contatadas para apresentação do projeto com até um mês de antecedência do início dos testes e aulas. Os pesquisadores foram muito bem recebidos pelas instituições e todo o apoio necessário foi dado por elas. Cada escola possuía pelo menos duas turmas de um ano específico (Escola A: duas turmas do quinto ano e Escola B: duas turmas de sexto ano). Uma das turmas selecionadas de maneira aleatória foi considerada uma Turma Experimental e a outra Turma de Controle. Ambas as turmas realizam as mesmas atividades, porém de maneira diferenciada. Um resumo das etapas da pesquisa encontra-se na Figura 2. As aulas com atividades de PC Desplugado são aplicadas na turma experimental após o pré-teste e antes do pós-teste, totalizando 10 horas aula. Para que a turma de controle tenha a mesma oportunidade de se beneficiar das atividades, as aulas são ministradas posteriormente, porém sem a geração de dados estatísticos.

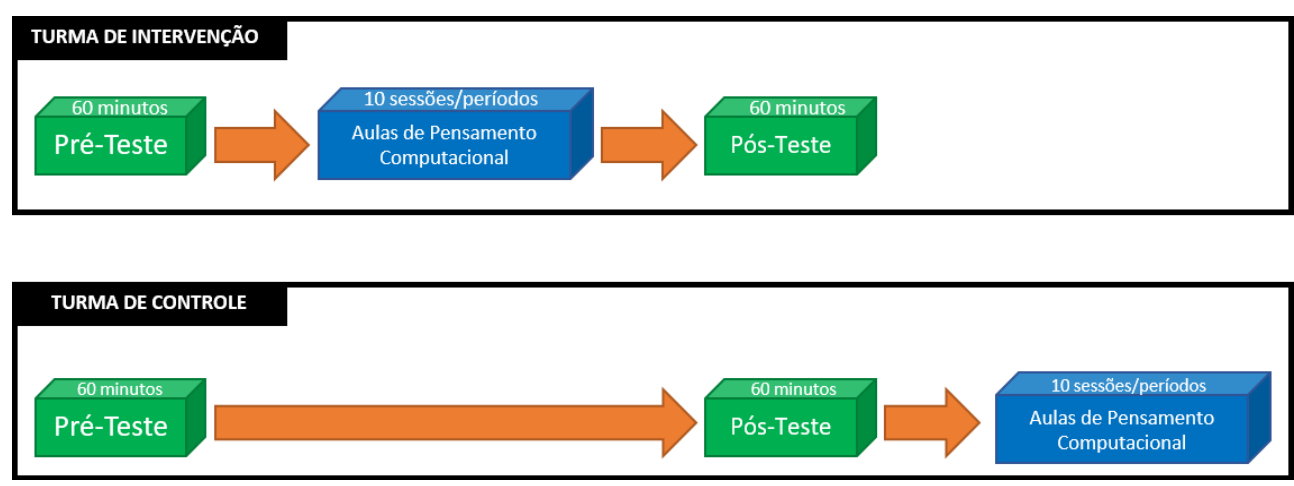

Figura 2 - Etapas da pesquisa

Na primeira semana da pesquisa, os alunos das quatro turmas foram convidados a participar do experimento, como parte das suas aulas regulares durante o primeiro semestre de 2017. Para aplicação dos pré-testes, os alunos foram acompanhados de suas professoras ao laboratório de informática da escola, onde permaneceram por até 60 minutos para a realização de um teste individual de Pensamento Computacional 
VI Congresso Brasileiro de Informática na Educação (CBIE 2017)

Anais dos Workshops do VI Congresso Brasileiro de Informática na Educação (WCBIE 2017)

desenvolvido por Román-González et al. (2015). Durante as próximas cinco semanas, foram ministradas aproximadamente duas horas semanais de aula de PC Desplugado no grupo experimental. Alguns dos materiais utilizados durante as aulas foram apresentados na Seção 3.3. Durante cada uma das sessões semanais, era possível trabalhar em média duas atividades. Na sétima semana, os estudantes de ambos os grupos (experimental e controle) foram novamente levados até o laboratório de informática para nova realização do teste. As respostas de todos os estudantes foram registradas na Google Cloud para que, posteriormente, pudessem ser visualizadas, recuperadas e convertidas. As respostas foram então tabuladas e analisadas estatisticamente. Os resultados e discussões sobre os dados coletados encontram-se disponíveis na próxima seção.

\section{Resultados e Discussões}

Esta seção apresenta os resultados dos Testes de Pensamento Computacional e uma breve discussão dos mesmos. Relembra-se que nenhum dos alunos teve contato com aulas formais de programação. A pontuação dos testes de PC é calculada de acordo com a quantidade de questões respondidas corretamente, lembrando que o teste é composto de 28 questões (pontuação máxima). O teste foi aplicado tanto no Grupo Experimental como no de Grupo Controle. Na Tabela 2 são apresentados os resultados das médias e desvios padrão (DP) das duas turmas e na Figura 3 o gráfico compara as pontuações entre o pré e pós-teste.

Tabela 2. Resultados Estatísticos

\begin{tabular}{l|c|c|c|c|c}
\hline \multicolumn{7}{|c}{ CONTROLE } \\
& N & $\begin{array}{c}\text { Pré-Teste } \\
\text { Média (DP) }\end{array}$ & $\begin{array}{c}\text { Pós-Teste } \\
\text { Média (DP) }\end{array}$ & p-valor & $\begin{array}{c}\text { Alteração de } \\
\text { Desempenho (\%) }\end{array}$ \\
\hline Desempenho & 37 & $10,27( \pm 3,26)$ & $10,84( \pm 3,62)$ & 0,267 & $+\mathbf{0 , 5 7}(\mathbf{5 , 5 5 \% )}$ \\
\hline \multicolumn{7}{|c}{ EXPERIMENTAL } \\
\hline & $\mathrm{N}$ & $\begin{array}{c}\text { Pré-Teste } \\
\text { Média (DP) }\end{array}$ & $\begin{array}{c}\text { Pós-Teste } \\
\text { Média (DP) }\end{array}$ & p-valor & $\begin{array}{c}\text { Alteração de } \\
\text { Desempenho (\%) }\end{array}$ \\
\hline Desempenho & 35 & $11,46( \pm 2,98)$ & $13,83( \pm 3,01)$ & $<0,001$ & $\mathbf{+ 2 , 3 7 ~ ( 2 0 , 6 8 \% )}$ \\
\hline
\end{tabular}

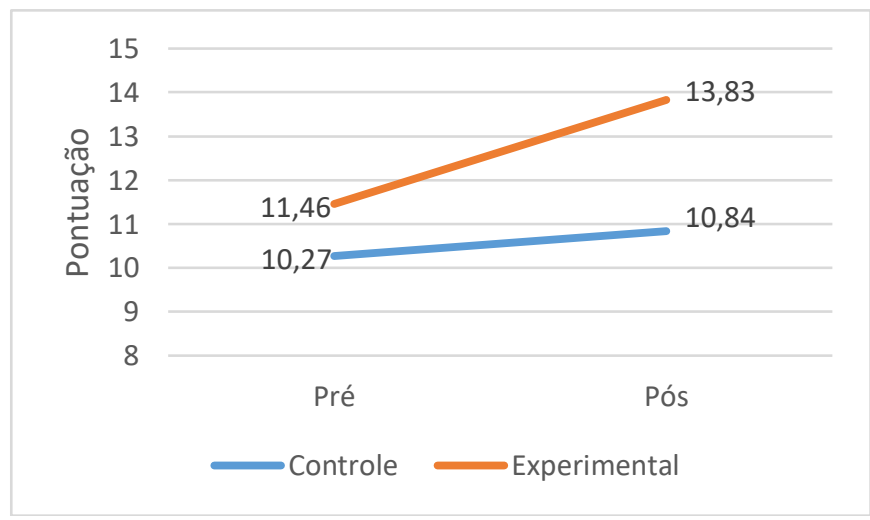

Figura 3. Gráfico comparativo dos resultados

Os resultados obtidos com este instrumento foram submetidos a procedimentos estatísticos a fim de testar a hipótese nula, ou seja, avaliar se houve diferença entre os resultados do pré-teste e pós-teste e se esta diferença era significativa. 
Para verificar se a melhora da média é estatisticamente significativa ou ocorreu de forma aleatória, foi utilizado o Teste T para amostras pareadas, considerando o intervalo de confiança de 95\%. No grupo onde houve intervenção (experimental), houve uma melhoria de $20,68 \%$ na quantidade de acertos e a análise estatística demonstrou um $\mathrm{P}$ $(\mathrm{T}<=\mathrm{t})$ bicaudal $<0,001$. Considerando que este valor é menor que 0,05 , comprova-se que há diferença significativa entre as médias, do ponto de vista estatístico. O mesmo tratamento de dados foi utilizado no grupo de controle, onde houve uma melhoria de apenas $0,57 \%$ de acertos e um $\mathrm{P}(\mathrm{T}<=\mathrm{t})$ bicaudal $=0,267$, ou seja, neste grupo não houve melhora do desempenho. Este dado reforça a teoria que o incremento do Pensamento Computacional no Grupo Experimental foi de fato motivado pelas atividades de PC Desplugado. Sendo assim, os dados apresentados demonstram que houve uma clara melhora no desempenho dos estudantes no grupo experimental e uma estagnação do grupo de controle, comprovando a hipótese inicial da pesquisa.

\section{Conclusões e Trabalhos Futuros}

Este artigo apresentou uma breve introdução do Pensamento Computacional, seu histórico e o estado-da-arte. Em seguida foram explanadas todas as etapas da pesquisa, os grupos participantes, o teste utilizado, os materiais desenvolvidos e os resultados.

Baseado na experiência adquirida durante o processo, pode-se concluir o seguinte:

- As crianças se mostraram muito entusiasmadas e motivadas durante as aulas de PC. Os professores também demonstraram muita satisfação com a oportunidade dada a seus alunos. De uma maneira geral, as escolas estão notavelmente receptivas com relação às aulas de $\mathrm{PC}$, não criando qualquer barreira para $\mathrm{o}$ projeto;

- Os dados positivos podem também ser compreendidos como uma variação muito pequena na melhoria de desempenho, porém é essencial considerar que ocorreram apenas 10 horas-aula de atividades desplugadas;

- Como pode ser visto na Tabela 2, houve uma melhora considerável na pontuação dos estudantes com resultados estatísticos altamente significativos no grupo experimental após apenas 10 horas de aula de Pensamento Computacional Desplugado, diferentemente do grupo de controle que manteve uma pontuação do pós-teste muito próxima do pré-teste. Esses resultados comprovam a eficácia da abordagem desplugada e atinge o objetivo desta investigação.

A abordagem desplugada tem suas limitações e, por isso, recomenda-se seu uso na introdução do Pensamento Computacional. Como trabalho futuro, propõe-se uma pesquisa mais detalhada para identificar o ponto de convergência da abordagem plugada e desplugada ou quando a abordagem desplugada perde sua eficácia e se torna necessário migrar para as máquinas. Outra proposta seria uma validação do Teste de PC no formato impresso.

Atualmente vemos um grande interesse e apoio de diversas instituições (públicas e privadas) para a adoção do PC na Educação Básica, porém é essencial divulgar sua importância, formar novos ou os atuais professores e cobrar dos legisladores para que estejam cientes disso. 


\section{Agradecimentos}

Esta pesquisa recebeu apoio financeiro da Agência Executiva de Educação, Audiovisual e Cultura da Comissão Europeia (EACEA / Programa SMART ${ }^{2}$ ) e Ministério da Educação (MEC) no Brasil. Os pesquisadores agradecem os colégios CEIP República de Ecuador school and CEIP Lope de Vega (Madrid, Espanha) por permitir a execução da investigação em suas instituições. Agradecemos também aos Estúdios Maurício de Souza S. A. e Mark Engelman (Thinkfun, Inc.) em permitir formalmente o uso de suas criações nas atividades.

\section{Referências}

ASSOCIATION FOR COMPUTING MACHINERY. A Model Curriculum for K-12 Computer Science: Final Report of the ACM K-12 Task Force Curriculum Committee. New York: ACM, 2003.

BBC LEARNING, B. What is computational thinking? , 2015. Disponível em: $<$ http://www.bbc.co.uk/education/guides/zp92mp3/revision>. Acesso em: 23/11/2015.

BELL, T.; WITTEN, I. H.; FELLOWS, M. Computer Science Unplugged... - Off-line activities and games for all ages (draft). $1^{\circ} \mathrm{ed} .1997$.

BELL, T.; WITTEN, I. H.; FELLOWS, M. Computer Science Unplugged. , 2015. Disponível em: <http://csunplugged.org/wpcontent/uploads/2015/03/CSUnplugged_OS_2015_v3.1.pdf >. Acesso em: 1/1/2015.

BRACKMANN, C.; BARONE, D.; CASALI, A.; BOUCINHA, R.; MUNOZHERNANDEZ, S. Computational thinking: Panorama of the Americas. . p.1-6, 2016. IEEE. Disponível em: <http://ieeexplore.ieee.org/document/7751839/>. Acesso em: $21 / 5 / 2017$.

CAMPOS, G.; CAVALHEIRO, S.; FOSS, L.; et al. Organização de Informações via Pensamento Computacional: Relato de Atividade Aplicada no Ensino Fundamental. 20o. Workshop de Informática na Escola (WIE 2014), Congresso Brasileiro de Informática na Educação - CBIE 2014., 2014.

CODE.ORG. Instructor Handbook - Code Studio Lesson Plans for Courses One, Two, and Three. CODE.ORG, 2015.

CURZON, P. cs4fn and computational thinking unplugged. . p.47-50, 2013. ACM Press. Disponível em: <http://dl.acm.org/citation.cfm?doid=2532748.2611263>. Acesso em: 20/5/2017.

CURZON, P.; MCOWAN, P. W.; PLANT, N.; MEAGHER, L. R. Introducing teachers to computational thinking using unplugged storytelling. . p.89-92, 2014. ACM Press. Disponível em: <http://dl.acm.org/citation.cfm?doid=2670757.2670767〉. Acesso em: 20/5/2017.

ENGELBERG, M.; THINKFUN. Code Master Programming Logic Game. , 2015. Thinkfun. Disponível em: <http://www.thinkfun.com/products/code-master/>. .

FEASTER, Y.; SEGARS, L.; WAHBA, S. K.; HALLSTROM, J. O. Teaching CS unplugged in the high school (with limited success). . p.248, 2011. ACM Press. Disponível em: <http://portal.acm.org/citation.cfm?doid=1999747.1999817〉. Acesso em: 20/5/2017. 
VI Congresso Brasileiro de Informática na Educação (CBIE 2017)

Anais dos Workshops do VI Congresso Brasileiro de Informática na Educação (WCBIE 2017)

FRANKLIN, D.; SKIFSTAD, G.; ROLOCK, R.; et al. Using Upper-Elementary Student Performance to Understand Conceptual Sequencing in a Blocks-based Curriculum. . p.231-236, 2017. ACM Press. Disponível em: <http://dl.acm.org/citation.cfm?doid=3017680.3017760>. Acesso em: 16/4/2017.

GROVER, S.; BASU, S. Measuring Student Learning in Introductory Block-Based Programming: Examining Misconceptions of Loops, Variables, and Boolean Logic. . p.267-272, 2017. ACM Press. Disponível em: <http://dl.acm.org/citation.cfm?doid=3017680.3017723>. Acesso em: 12/7/2017.

GROVER, S.; PEA, R. Computational Thinking in K-12: A Review of the State of the Field. Educational Researcher, v. 42, n. 1, p. 38-43, 2013.

KOLOGESKI, A.; SILVA, C.; BARBOSA, D.; MATTOS, R.; MIORELLI, S. Desenvolvendo o Raciocínio Lógico e o Pensamento Computacional: Experiências no Contexto do Projeto Logicando. Revista Novas Tecnologias na Educação (RENOTE), v. 14, n. 2, 2016.

LAMBERT, L.; GUIFFRE, H. Computer science outreach in an elementary school. Journal of Computing Sciences in Colleges, v. 24, n. 3, p. 118-124, 2009.

LIUKAS, L. Hello Ruby: adventures in coding. Feiwel \& Friends, 2015.

NISHIDA, T.; KANEMUNE, S.; IDOSAKA, Y.; et al. A CS Unplugged Design Pattern. p.231-235, 2009. New York, NY: ACM. Disponível em: <http://dx.doi.org/10.1145/1508865>. Acesso em: 18/5/2017.

RODRIGUEZ, B.; STEPHEN, K.; RADER, C.; CAMP, T. Assessing Computational Thinking in CS Unplugged Activities. Proceedings of the 2017 ACM SIGCSE Technical Symposium on Computer Science Education. p.501-506, 2017. Seattle, Washington, USA: ACM.

ROMÁN-GONZÁLEZ, M. Computational Thinking Test: Design Guidelines and Content Validation. Proceedings of the 7th Annual International Conference on Education and New Learning Technologies (EDULEARN 2015). p.2436-2444, 2015. Barcelona, Spain: IATED.

ROMÁN-GONZÁLEZ, M. Codigoalfabetización y Pensamiento Computacional en Educación Primaria y Secundaria: Validación de un Instrumento y Evaluación de Programas, fev. 2016. Madrid, Spain: Universidad Nacional de Educación a Distancia.

ROMÁN-GONZÁLEZ, M.; PÉREZ, J. C.; CARMEN JIMÉNEZ-FERNÁNDEZ. Test de Pensamiento Computacional: diseño y psicometría general, 2015. Disponível em: <https://doi.org/10.13140/RG.2.1.3056.5521>. Acesso em: 16/4/2017.

SCAICO, P.; MYCHELliNE, H.; CUNHA, F.; ALENCAR, Y. Um Relato de Experiências de Estagiários da Licenciatura em Computação com o Ensino de Computação para Crianças. Revista Novas Tecnologias na Educação (RENOTE), v. 10, n. 3, 2012.

TAUB, R.; BEN-ARI, M.; ARMONI, M. The effect of CS unplugged on middle-school students' views of CS. . p.99, 2009. ACM Press. Disponível em: <http://portal.acm.org/citation.cfm?doid=1562877.1562912>. Acesso em: 20/5/2017.

WING, J. M. Computational thinking. Communications of the ACM, v. 49, n. 3, p. 33, 2006. 